\title{
Semicarbazone and Thiosemicarbazone of 5-acetyl-3-(methylsulfanyl)-1,2,4-triazine
}

\section{Mariusz Mojzych}

Institute of Chemistry, University of Podlasie, ul. 3 Maja 54, 08-110 Siedlce, Poland

e-mail: mojzych@ap.siedlce.pl

Received: 11 May 2004 / Accepted: 30 May 2004 / Published: 1 October 2005

Keywords: semicarbazone, thiosemicarbazone, 5-acetyl-1,2,4-triazine.

Semicarbazone as well as thiosemicarbazone of 5-acetyl-3-(methylsulfanyl)-1,2,4-triazine were synthesised as reactive intermediates for the synthesis of $1 H$-pyrazolo[4,3-e $][1,2,4]$ triazine derivatives via acid promoted ring closure [1-3].
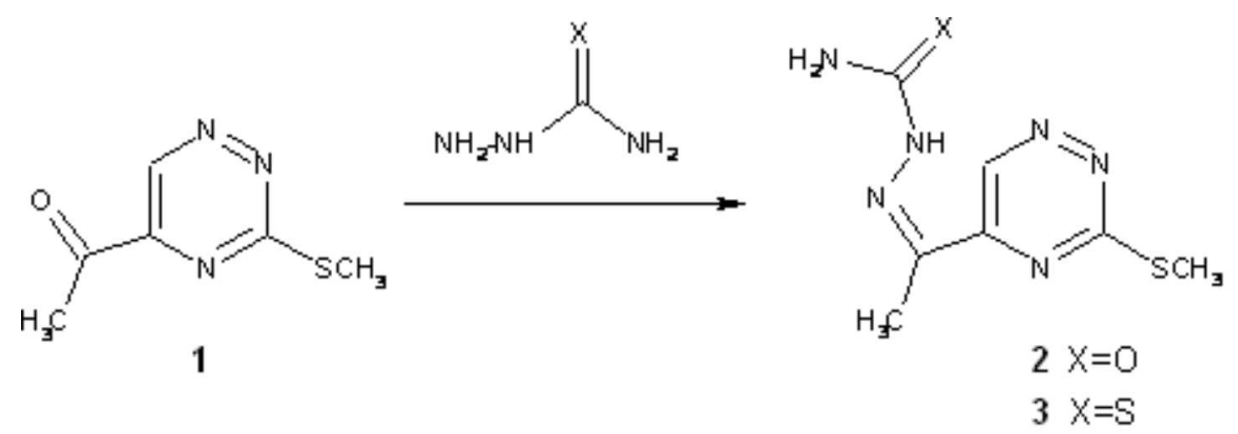

Starting ketone 1 was prepared according to a reported procedure [4]. To a solution of ketone $\mathbf{1}$ (169 mg, $1 \mathrm{mmol})$ and semicarbazone or thiosemicarbazone $(1.2 \mathrm{mmol})$ in ethanol $(20 \mathrm{ml}) 10 \% \mathrm{HCl}(0.5 \mathrm{ml}) \mathrm{was}$ added. The resulting reaction mixture was heated at reflux for $5 \mathrm{~min}$ and then stirred at room temperature for additional $30 \mathrm{~min}$. The precipitated solid was filtered off, recrystallized from dioxane and dried under vacuum.

\section{Semicarbazone of 5-acetyl-3-(methylsulfanyl)-1,2,4-triazine (2)}

Yield 89\%.

Melting Point: $260^{\circ} \mathrm{C}$.

${ }^{1} \mathrm{H}-\mathrm{NMR}\left(200 \mathrm{MHz}, \mathrm{DMSO}-d_{6}\right): \delta=2.19(\mathrm{~s}, 3 \mathrm{H}) ; 2.62(\mathrm{~s}, 3 \mathrm{H}) ; 6.96\left(\mathrm{~s}, 2 \mathrm{H}, \mathrm{NH}_{2}\right) ; 9.98(\mathrm{~s}, 1 \mathrm{H}) ; 10.13(\mathrm{~s}$, $1 \mathrm{H}, \mathrm{NH})$.

IR $\left(\mathrm{CHCl}_{3}\right.$ film, $\left.\mathrm{cm}^{-1}\right): 3339 ; 3106 ; 1693 ; 1520 ; 1486 ; 1426 ; 1312 ; 1256 ; 1183 ; 1133 ; 1058 ; 871 ; 713$.

MS- EI (m/z, \%): $226(18)\left[\mathrm{M}^{+}\right] ; 209$ (7); 184 (11); 183 (100); 182 (62); 155 (12); 154 (39); 140 (32); 82 (49); 81 (30); 74 (18); 53 (19).

HR-MS: Calculated for $\mathrm{C}_{7} \mathrm{H}_{10} \mathrm{~N}_{6} \mathrm{OS}: 226.0636$. Found: 226.06314.

Thiosemicarbazone of 5-acetyl-3-(methylsulfanyl)-1,2,4-triazine (3)

Yield 87\%. 
Melting Point: $229^{\circ} \mathrm{C}$.

IR $\left(\mathrm{CHCl}_{3}\right.$ film, $\left.\mathrm{cm}^{-1}\right): 3304 ; 3158 ; 2969 ; 1587 ; 1495 ; 1423 ; 1243 ; 1127 ; 1057 ; 876$.

${ }^{1} \mathrm{H}-\mathrm{NMR}\left(200 \mathrm{MHz}, \mathrm{DMSO}-d_{6}\right): \delta=2.31(\mathrm{~s}, 3 \mathrm{H}) ; 2.63(\mathrm{~s}, 3 \mathrm{H}) ; 8.53(\mathrm{~s}, 1 \mathrm{H}) ; 8.70(\mathrm{~s}, 1 \mathrm{H}) ; 10.08(\mathrm{~s}, 1 \mathrm{H})$; $10.81(\mathrm{~s}, 1 \mathrm{H})$.

MS-EI (m/z, \%): $242(80)\left[\mathrm{M}^{+}\right] ; 199(21) ; 167(7) ; 154(6) ; 141(5) ; 140(22) ; 128(27) ; 116(100) ; 82$ (12); 81 (12); 74 (14); 60 (24); 53 (13).

HR-MS: Calculated for $\mathrm{C}_{7} \mathrm{H}_{10} \mathrm{~N}_{6} \mathrm{~S}_{2}$ : 242.04084; Found: 242.04129.

\section{References:}

1.Rykowski, A.; Mojzych, M.; Karczmarzyk, Z. Heterocycles, 2000, 53, 2175.

2.Mojzych, M.; Rykowski, A. Polish J. Chem., 2003, 77, 1797.

3.Mojzych, M.; Rykowski, A. Heterocycles, 2004, submitted.

4.Rykowski, A.; Lipińska, T. Synth. Comm., 1996, 26, 4409.

Sample Availability: Available from MDPI.

(C) 2005 MDPI. All rights reserved. 